\title{
Interpolation d'opérateurs entre espaces de fonctions holomorphes
}

\author{
par PATRICE LAssere (Toulouse)
}

\begin{abstract}
Let $K$ be a compact subset of an hyperconvex open set $D \subset \mathbb{C}^{n}$, forming with $D$ a Runge pair and such that the extremal p.s.h. function $\omega(\cdot, K, D)$ is continuous. Let $H(D)$ and $H(K)$ be the spaces of holomorphic functions respectively on $D$ and $K$ equipped with their usual topologies. The main result of this paper contains as a particular case the following statement: if $T$ is a continuous linear map of $H(K)$ into $H(K)$ whose restriction to $H(D)$ is continuous into $H(D)$, then the restriction of $T$ to $H\left(D_{\alpha}\right)$ is a continuous linear map of $H\left(D_{\alpha}\right)$ into $\left.H\left(D_{\alpha}\right), \forall \alpha \in\right] 0,1\left[\right.$ where $D_{\alpha}=\{z \in D: \omega(z, D, K)<\alpha\}$.
\end{abstract}

1. Introduction. Il y a environ 30 ans, M. Zerner a démontré (manuscrit non publié) le résultat suivant : Si $T$ est un opérateur linéaire continu de $H(D(0, r)$ ) (ou $H(\overline{D(0, r)})$, avec $D(0, r)=\{z \in \mathbb{C}:|z|<r\}$, dans $H(D(0, r))$ tel que sa restriction à $H(D(0, R))(0<r<R<\infty)$ soit encore linéaire continue à valeurs dans $H(D(0, R))$, alors pour tout $\tau(r<\tau<$ $R)$, la restriction de $T$ à $H(D(0, \tau))$ est un opérateur linéaire continue de $H(D(0, \tau))$ dans lui-même. Ce résultat a été retrouvé un peu plus tard d'une manière indépendante par H. Mascart ([MA], p. 19).

Dans un article paru en 1964 J. L. Lions et J. Peetre ([L.P.]) s'intéressent à une certaine classe d'espaces intermédiaires possédant la propriété d'interpolation par rapport aux applications linéaires continues. Autrement dit : Si $A$ est construit à partir du couple $\left(A_{0}, A_{1}\right)$ et si $B$ est obtenu par la même construction à partir du couple $\left(B_{0}, B_{1}\right)$, alors toute application linéaire continue de $A_{i}$ dans $B_{i}(i=0$ et 1$)$ applique continûment $A$ dans $B$. Plus particulièrement, ils retrouvent le résultat de Zerner.

En 1972, Nguyen Thanh Van ([N], Chap. IV, §2) montre que les espaces intermédiaires $\left(H\left(D_{\alpha}\right)\right)_{0<\alpha<1}$ au couple $(H(K), H(D)$ ) ( $K$ étant un compact du domaine $D$ de $\mathbb{C}$, tous deux satisfaisant à certaines propriétés; les

1991 Mathematics Subject Classification: 32A10, 32F05, 46A12, 46E10.

Key words and phrases: interpolation of operators, spaces of analytic functions, common Schauder bases. 
$D_{\alpha}$ étant les ensembles de niveau de la mesure harmonique de la frontière de $D$ par rapport à $D \backslash K)$ coïncident avec les espaces d'interpolation de Lions-Peetre du couple $(H(\stackrel{\circ}{K}), H(D))$. En particulier, on a une première généralisation du résultat de Zerner.

Nous proposons ici une nouvelle extension dans le cadre pluricomplexe et avec des familles équicontinues d'applications linéaires. Enfin, comme application nous retrouvons un théorème de prolongement des bases communes de Zakharyuta.

\section{Préliminaires}

2.1. Si $K$ est un compact de $\mathbb{C}^{n}, H(K)$ désignera l'espace des germes de fonctions analytiques sur $K$ muni de sa topologie usuelle : $H(K)=$ $\lim _{\text {.ind }}{ }_{D K K} H(D), D$ parcourant la famille des voisinages ouverts de $K$, $H(D)$ étant l'espace des fonctions analytiques sur $D$ muni de la topologie de la convergence compacte.

Pour un ouvert $D$ de $\mathbb{C}^{n}$ et un compact $K$ de $D$ nous dirons que $D$ est un voisinage de Runge de $K$ si $H(D)$ est dense dans $H(K)$. Enfin, $P S H(D)$ sera le cône des fonctions plurisousharmoniques sur $D$.

2.2. Dans toute la suite $D$ sera un ouvert hyperconvexe de $\mathbb{C}^{n}$, i.e. il existe sur $D$ une fonction plurisousharmonique d'exhaustion, continue et négative. Pour un compact $K$ de $D$ dont l'intersection avec chaque composante connexe de $D$ est non pluripolaire on définit la fonction extrémale du couple $(K, D)$ par

$$
\omega(z, K, D):=\sup \{u(z): u \in P S H(D), u \leq 1, u \mid K \leq 0\} .
$$

Le couple $(K, D)$ sera dit $P$-régulier si sa fonction extrémale $\omega(\cdot, K, D)$ est continue sur $D$.

Sous ces hypothèses et si, pour $\alpha \in] 0,1[$,

$$
D_{\alpha}:=\{z \in D: \omega(z, K, D)<\alpha\}, \quad F_{\alpha}:=\{z \in D: \omega(z, K, D) \leq \alpha\},
$$

on a les propriétés suivantes :

(1) Pour $0 \leq \beta<\alpha \leq 1$

$$
\omega\left(z, F_{\alpha}, D_{\alpha}\right)= \begin{cases}\frac{\omega(z, K, D)-\beta}{\alpha-\beta} & \text { si } z \in D_{\alpha} \backslash F_{\beta}, \\ 0 & \text { si } z \in F_{\beta} .\end{cases}
$$

(2) Si $\widehat{K}_{D}:=\left\{z \in D:|f(z)| \leq\|f\|_{K}, \forall f \in H(D)\right\}$, alors

$$
\bigcap_{0<\alpha<1} D_{\alpha}=\widehat{K}_{D}, \quad \bigcup_{0<\alpha<1} D_{\alpha}=D
$$


En particulier, la topologie de $H(D)$ est engendrée par la famille de seminormes $\left(P_{\alpha}\right)_{0<\alpha<1}$ où $P_{\alpha}(f):=|f|_{D_{\alpha}}:=\sup _{z \in D_{\alpha}}|f(z)|$ et l'on a

$H\left(\widehat{K}_{D}\right)=\lim _{\alpha \rightarrow 0} \operatorname{ind} H\left(D_{\alpha}\right)=\lim _{\alpha \rightarrow 0} H^{\infty}\left(D_{\alpha}\right), \quad H(D)=\lim _{\alpha \rightarrow 1} \operatorname{proj} H\left(D_{\alpha}\right)$,

$H^{\infty}\left(D_{\alpha}\right)$ désignant l'espace des fonctions holomorphes et bornées sur $D_{\alpha}$.

(3) Si de plus $D$ est un voisinage de Runge de $K$ alors $H(K) \simeq H\left(\widehat{K}_{D}\right)$.

Pour plus de précision consulter par exemple [ZA-1].

2.3. L'opérateur de Monge-Ampère complexe permet de définir sur $D$ une mesure de Radon positive $\lambda_{0}:=\left(d d^{c} \omega^{*}(\cdot, K, D)\right)^{n}\left(\omega^{*}\right.$ désignant la régularisée semi-continue supérieurement de $\omega)$ dont la masse totale est la capacité de $K$ relative à $D$. $K$ étant ici compact, la mesure est alors concentrée sur $K$ et s'identifie à une mesure de Radon positive portée par $K$ (cf. [B.T.]).

Nous utiliserons dans ce travail des résultats récents d'Ahmed Zeriahi ([ZE]) que nous regroupons dans l'énoncé suivant :

Proposition 2.1. Sous les hypothèses du paragraphe 2.3 il existe sur $D$ une mesure $d \lambda_{1}$ telle que :

Si $H_{0}$ est le sous-espace fermé de $L^{2}\left(K, d \lambda_{0}\right)$ engendré par les restrictions à $K$ des fonctions holomorphes au voisinage de $K$ et $H_{1}:=H(D) \cap$ $L^{2}\left(D, d \lambda_{1}\right)$, alors le système doublement orthogonal $\left\{\varphi_{n}\right\}$ dans $H_{0}$ et $H_{1}$ construit suivant la méthode de Bergman ([BE]) est une base commune des espaces $H(K), H(D)$ et $H\left(D_{\alpha}\right)(0<\alpha<1)$ ayant les propriétés suivantes:

$$
\begin{gathered}
\lim _{n \rightarrow+\infty} \frac{\log \left|\varphi_{n}\right| D_{\alpha}}{\log \mu_{n}}=\alpha, \quad \text { où } \quad \mu_{n}:=\left(\int_{D}\left|\varphi_{n}\right|^{2} d \lambda_{1}\right)^{1 / 2} . \\
\sum_{n=0}^{+\infty} \mu_{n}^{-\delta}<\infty, \quad \forall \delta>0 .
\end{gathered}
$$

(4-3) Si $f \in H\left(D_{\alpha}\right)$ alors pour tout $\left.\varepsilon \in\right] 0, \alpha[$ il existe une constante $M(f, \varepsilon, \alpha)$ telle que

$$
\left|\varphi_{n}^{*}(f)\right| \leq M(f, \alpha, \varepsilon) \mu_{n}^{-\alpha+\varepsilon}, \quad \forall n \in \mathbb{N},
$$

$\left\{\varphi_{n}^{*}(f)\right\}$ désignant la suite des coefficients de Fourier de $f$ par rapport à la base $\left\{\varphi_{n}\right\}$.

3. Résultat principal. On note par $\Omega$ (resp. $D$ ) un ouvert hyperconvexe de $\mathbb{C}^{n}$ (resp. $\left.\mathbb{C}^{m}\right)$ et $\chi($ resp. $K)$ un compact de $\Omega$ (resp. $D$ ) tel que le couple $(\chi, \Omega)$ (resp. $(K, D))$ soit $P$-régulier et forme une paire de Runge.

Proposition 3.1. Soit $\mathcal{T}$ une famille équicontinue d'applications linéaires de $H(\chi)$ dans $H(K)$ dont la restriction à $H(\Omega)$ est une famille 
équicontinue de $H(\Omega)$ dans $H(D)$. Alors pour tout $\alpha$ dans ]0, $1[$ la restriction de $\mathcal{T}$ à $H\left(\Omega_{\alpha}\right)$ est une famille équicontinue d'applications linéaires de $H\left(\Omega_{\alpha}\right)$ dans $H\left(D_{\alpha}\right)$.

Preuve. Nous allons procéder par étapes :

LEMME 3.1. Si $\mathcal{T}$ est une famille équicontinue d'applications linéaires de $H(\chi)$ dans $H(K)$, alors pour tout $\alpha \in] 0,1\left[\right.$ il existe une constante $C_{\alpha}>0$ telle que

$$
\|T(\widetilde{f})\|_{K} \leq C_{\alpha}\|f\|_{\Omega_{\alpha}}, \quad \forall f \in H^{\infty}\left(\Omega_{\alpha}\right), \forall T \in \mathcal{T}
$$

( $\widetilde{f}$ est le germe de $f$ dans $H(K)$.)

Preuve. C'est une conséquence immédiate de $H(K)=\lim _{\text {.ind }}{ }_{\alpha \rightarrow 0}$ $H^{\infty}\left(\Omega_{\alpha}\right)$ et du fait que \|\|$_{K}$ est une semi-norme continue sur $H(K)$.

Dorénavant, pour plus de simplicité $f$ représentera aussi bien la fonction analytique que son germe.

Soit alors la base $\left\{\varphi_{n}\right\}$ de la proposition 2.1. D'après le lemme 3.1 on a

$$
\left\|T\left(\varphi_{n}\right)\right\|_{K} \leq M_{\alpha}\left\|\varphi_{n}\right\|_{\Omega_{\alpha}}, \quad \forall n \in \mathbb{N}, \forall T \in \mathcal{T} .
$$

Et de (4-1) résulte

$$
\left\|T\left(\varphi_{n}\right)\right\|_{K} \leq C_{\alpha} \mu_{n}^{\alpha}, \quad \forall n \in \mathbb{N}, \forall T \in \mathcal{T} .
$$

Par ailleurs $\mathcal{T}$ étant une famille équicontinue de $H(\Omega)$ dans $H(D)$, par (2) pour tout $\beta$ dans ]0,1[ il existe $\gamma \in] 0,1[$ et une constante $M(\beta)>0$ tels que

$$
\left\|T\left(\varphi_{n}\right)\right\|_{D_{\beta}} \leq M(\beta)\left\|\varphi_{n}\right\|_{\Omega_{\gamma}}, \quad \forall T \in \mathcal{T}, \forall n \in \mathbb{N} .
$$

En utilisant à nouveau (4-1) on obtient

(7) $\forall \beta \in] 0,1\left[\exists C_{\beta}>0 \quad\left\|T\left(\varphi_{n}\right)\right\|_{D_{\beta}} \leq C_{\beta} \mu_{n}, \quad \forall n \in \mathbb{N}, \forall T \in \mathcal{T}\right.$.

LEMME 3.2. Sous les hypothèses de la proposition 3.1 on a

$\forall \alpha \in] 0,1[\exists \varepsilon \in] 0,1-\alpha[$ et $M(\alpha, \varepsilon)>0$ tels que

$$
\left\|T\left(\varphi_{n}\right)\right\|_{D_{\alpha}} \leq M(\alpha, \varepsilon) \mu_{n}^{\alpha+\varepsilon}, \quad \forall n \in \mathbb{N}, \forall T \in \mathcal{T} .
$$

Preuve. Soit $\beta>\alpha$. Par (1) nous avons

$$
\omega\left(z, K, D_{\beta}\right)=\omega(z, K, D) / \beta,
$$

et donc pour $\beta \in] \alpha /(\alpha+\varepsilon / 2), 1[$

$$
D_{\alpha} \Subset \widetilde{D}_{\alpha+\varepsilon / 2}:=\left\{z \in D_{\beta}: \omega\left(z, K, D_{\beta}\right)<\alpha+\varepsilon / 2\right\} .
$$

Soit

$$
\left\|T\left(\varphi_{n}\right)\right\|_{D_{\alpha}} \leq\left\|T\left(\varphi_{n}\right)\right\|_{\tilde{D}_{\alpha+\varepsilon / 2}}, \quad \forall n \in \mathbb{N}, \forall T \in \mathcal{T}
$$

Il résulte alors du théorème des deux constantes généralisé que

$$
\left\|T\left(\varphi_{n}\right)\right\|_{D_{\alpha}} \leq\left\|T\left(\varphi_{n}\right)\right\|_{\tilde{D}_{\alpha+\varepsilon / 2}} \leq\left\|T\left(\varphi_{n}\right)\right\|_{K}^{1-(\alpha+\varepsilon / 2)}\left\|T\left(\varphi_{n}\right)\right\|_{D_{\beta}}^{\alpha+\varepsilon / 2},
$$


et des inégalités (6) et (7) :

$$
\left\|T\left(\varphi_{n}\right)\right\|_{D_{\alpha}} \leq M(\gamma) \mu_{n}^{\gamma(1-(\alpha+\varepsilon / 2))} \mu_{n}^{\alpha+\varepsilon / 2}
$$

où $\gamma \in] 0,1[$. Choisissons maintenant $\gamma$ tel que $0<\gamma(1-(\alpha+\varepsilon / 2))<\varepsilon / 2$; on obtient

$$
\left\|T\left(\varphi_{n}\right)\right\|_{D_{\alpha}} \leq M(\alpha, \varepsilon) \mu_{n}^{\alpha+\varepsilon} .
$$

Nous sommes maintenant en mesure de démontrer la proposition 3.1. Soit $\alpha \in] 0,1\left[\right.$ et $T \in \mathcal{T}$. Si $f \in H\left(\Omega_{\alpha}\right)$, par la proposition 2.1, $f=$ $\sum_{n=0}^{\infty} \varphi_{n}^{*}(f) \varphi_{n}$, avec convergence dans $H\left(\Omega_{\alpha}\right)$.

De (8), (4-3) et (4-2) résulte la normale convergence sur tout compact de $D_{\alpha}$ de la série $\sum_{n=0}^{\infty} \varphi_{n}^{*}(f) T\left(\varphi_{n}\right)$. Si on pose $T_{\alpha}(f):=\sum_{n=0}^{\infty} \varphi_{n}^{*}(f) T\left(\varphi_{n}\right)$, $T_{\alpha}$ ainsi définie est bien une application linéaire continue de $H\left(D_{\alpha}\right)$ dans $H\left(\Omega_{\alpha}\right)$ : c'est la restriction de $T$ à $H\left(\Omega_{\alpha}\right)$.

Par ailleurs, vu que dans $(8), M(\alpha, \varepsilon)$ est indépendante de $T$, il découle immédiatement que pour tout $f \in H\left(\Omega_{\alpha}\right),\left\{T_{\alpha}(f): T \in \mathcal{T}\right\}$ est une partie bornée de $H\left(D_{\alpha}\right)$.

Les hypothèses du théorème de Banach-Steinhaus sont réalisées et donc $\left\{T_{\alpha}: T \in \mathcal{T}\right\}$ est une famille équicontinue d'applications linéaires de $H\left(\Omega_{\alpha}\right)$ dans $H\left(D_{\alpha}\right)$.

\section{Application à un théorème de prolongement des bases com-} munes. La proposition 3.1 nous permet de redémontrer assez rapidement le résultat suivant dû à V. P. Zakharyuta ([ZA-2], th. 4.6).

Proposition 4.1. Soient $K$ et $D$ comme dans le paragraphe précédent. Si $\left\{\varphi_{n}\right\}$ est une base commune des espaces $H(K)$ et $H(D)$ alors elle est une base de $H\left(D_{\alpha}\right)$ pour tout $\alpha$ dans $] 0,1[$.

Preuve. $\left\{\varphi_{n}\right\}$ étant par hypothèse une base de $H(K)$, on note par $\left\{\varphi_{n}^{*}\right\}$ sa base duale; c'est une base de $H(K)^{*}$. Soit $\left.\alpha \in\right] 0,1\left[\right.$ et $f \in H\left(D_{\alpha}\right)$; on doit prouver qu'il existe une et une seule suite $\left\{C_{k}\right\}$ telle que

$$
\sum_{k=0}^{n} C_{k} \varphi_{k} \text { converge vers } f \text { dans } H\left(D_{\alpha}\right) \text { (quand } n \rightarrow+\infty \text { ). }
$$

Si $\left\{C_{k}\right\}$ vérifie (9), elle est unique : en effet, puisque $\left\{\varphi_{n}\right\}$ est une base de $H(K)$ et que $H\left(D_{\alpha}\right) \hookrightarrow H(K)$ on a $C_{k}=\varphi_{k}^{*}(f)$. Tout revient donc à montrer que $\sum_{k=0}^{n} \varphi_{k}^{*}(f) \varphi_{k}$ converge vers $f$ dans $H\left(D_{\alpha}\right)$. Pour tout $g \in$ $H(K)$ (resp. $H(D))$ on pose

$$
T_{n}(g):=\sum_{k=0}^{n} \varphi_{k}^{*}(g) \varphi_{k} .
$$

Par hypothèse $\lim _{n \rightarrow+\infty} T_{n}(g)=g$ dans $H(K)$ (resp. $\left.H(D)\right)$. 
En raison du théorème de Banach-Steinhaus $\left\{T_{n}\right\}$ est une famille équicontinue d'applications linéaires de $H(K)$ dans $H(K)$ (resp. de $H(D)$ dans $H(D)$ ). Donc, en raison de la proposition $3.1,\left\{T_{n}\right\}$ est aussi équicontinue de $H\left(D_{\alpha}\right)$ dans $H\left(D_{\alpha}\right)$.

De plus, pour tout $k \in \mathbb{N}, T_{n}\left(\varphi_{k}\right)=\varphi_{k}, \forall n \geq k$, et $\left\{\varphi_{k}\right\}$ est totale dans $H\left(D_{\alpha}\right)$, donc la suite $\left\{T_{n}\right\}$ converge simplement vers une application linéaire continue $T$ de $H\left(D_{\alpha}\right)$ dans $H\left(D_{\alpha}\right)$. T n'est autre que l'application identique, car elle coïncide avec celle-ci sur la suite totale $\left\{\varphi_{k}\right\}$, d'où la conclusion.

Remarque. Dans la terminologie de Zakharyuta ([ZA-1]) un ouvert hyperconvexe est appelé un ouvert fortement pseudoconvexe.

\section{Bibliographie}

[B.T.] E. Bedford and B. A. Taylor, A new capacity for plurisubharmonic functions, Acta Math. 149 (1982), 1-40.

[B] S. Bergman, The Kernel Function and Conformal Mapping, Math. Surveys 5, Amer. Math. Soc., 1970.

[L.P.] J. L. Lions et J. Peetre, Sur une classe d'espaces d'interpolation, Publ. Math. I.H.E.S. 19 (1964), 4-68.

[MA] H. Mascart, Sur quelques opérateurs linéaires différentiels, Ann. Fac. Sci. Toulouse 24 (1960), 5-73.

[N] Nguyen Thanh Van, Bases de Schauder dans certains espaces de fonctions holomorphes, Ann. Inst. Fourier (Grenoble) 22 (2) (1972), 169-253.

[ZA-1,2] V. P. Zakharyuta, Fonctions plurisousharmoniques extrémales, échelles hilbertiennes et isomorphismes d'espaces de fonctions analytiques de plusieurs variables complexes I, II, Teor. Funktsiu Funktsional. Anal. i Prilozhen. 19 (1974), 133-157; 21 (1974), 65-83 (en russe).

[ZE] A. Zeriahi, Bases de Schauder et isomorphismes d'espaces de fonctions holomorphes, C. R. Acad. Sci. Paris 310 (1990), 691-694.

LABORATOIRE D'ANALYSE COMPLEXE ET D'ANALYSE FONCTIONNELLE UNIVERSITÉ PAUL SABATIER

118, ROUTE DE NARBONNE

31062 TOULOUSE CEDEX, FRANCE

E-MAIL: LASSERE@FRCICT81.BITNET 